\title{
On the Efficient Resource Allocation for High Quality Video Streams and FTP Traffic over Next Generation Wireless Networks
}

\author{
Polychronis Koutsakis and Spyros Psychis \\ Department of Electronic and Computer Engineering, \\ Technical University of Crete, 73100 Chania, Greece \\ \{polk, psycho\}atelecom.tuc.gr
}

\begin{abstract}
In this paper we propose a Medium Access Control (MAC) protocol for the efficient integration of high quality video traffic with ftp data packet traffic over a wireless channel of very high capacity. Via an extensive simulation study, we evaluate the system's performance under a variety of possible loads which consist of actual MPEG-4 streams and ftp sessions.
\end{abstract}

\section{Introduction}

Within a microcell, spatially dispersed mobile terminals (MTs) share a radio channel that connects them to a fixed Base Station (BS). Fixed-length packets arriving at the mobiles are buffered at the terminals until they are transmitted on the uplink to the BS. The BS allocates channel resources, delivers feedback information and transfers the packets to the wired networks and the Internet. In this work, MTs are considered to be high performance devices with extended storage capabilities which can act like cache memories, streaming multimedia material (video, data) to other MTs in the same or in adjacent microcells, in order to reduce client start-up latencies and improve network performance. Therefore, we envision high quality stored video streaming on the uplink channel (MTs to the BS) in order for these streams to be delivered for playback to their destinations.

The delivery of high quality stored video material is a service with very strict QoS requirements. Consequently, the design of a MAC scheme which integrates this class of service with others, often requiring contradictory QoS guarantees, is necessary. In this study we design and evaluate a MAC scheme for the uplink wireless channel, as in the downlink channel the BS is the sole transmitter, and therefore is in complete control of the downstream traffic.

\section{Traffic Models}

In our study, we use the trace statistics of actual MPEG-4 streams from [1]. The video streams (Silence of the Lambs, The Simpsons, Soccer, Star Wars, Parking Lot Camera) were carefully selected in order to cover a broad range of movie characteristics. New video frames are generated every 40 msecs. We have made the assumption that all packets of a video frame (VF) must be delivered before the next VF arrives. The allowed video packet dropping probability is set to 0.0001 [2]. 
Regarding data traffic, we use the web traffic model presented in [3], the parameters of which result in an average web request size of about $50 \mathrm{~KB}$. As in the reference model of UMTS [4], an FTP file transfer can be described as a web session with only one web request. The arrival process of FTP sessions is Poisson with rate $\lambda$ sessions per second. An upper bound of one minute is set on the average ftp session delay as a user QoS requirement. This is not a strict requirement, given that the average ftp session size of $50 \mathrm{~KB}$ needs in our system a mere $2 \%$ average use of the channel information bandwidth per frame, in order to satisfy the specific bound.

\section{Frame structure, Base Station Scheduling, Actions of the Mobile Terminals and System Parameters}

The uplink channel time is divided into time frames of equal length. Each frame consists of two types of intervals. These are the request intervals and the information intervals. Each of these intervals is divided into a number of time slots.

Within an information interval, each slot accommodates exactly one, fixed length, packet that contains video or ftp data information and a header. Each request slot is subdivided into two mini-slots and each mini-slot accommodates exactly one, fixed length, request packet. By using more than one minislot per request slot, a more efficient usage of the available request bandwidth is possible [2]. The base station broadcasts a short binary feedback packet at the end of each mini-slot indicating only the presence or absence of a collision within the mini-slot (collision (C) versus noncollision (NC)). Upon successfully transmitting a request packet the terminal waits until the end of the corresponding request interval to learn of its reservation slot (or slots). If unsuccessful within the request intervals of the current frame, the terminal attempts again in the request intervals of the next frame.

Video and ftp terminals share the request slots. The two-cell stack reservation random access algorithm [5] is used to resolve the collisions, among video request packets. After the end of the video contention period, the two-cell stack random access algorithm [5] is used to resolve the collisions among ftp terminals with sessions ready for transmission, due to its operational simplicity, stability and high throughput.

When a video terminal wants to decrease its bit rate, it releases all the slots that were previously allocated to it and are no longer necessary. The BS consequently allocates the newly released slots to any other requesting terminals, after the end of the request interval of the next frame. When the bit rate of a video terminal increases, then the terminal must enter the contention process in order to acquire the additional information slots it needs.

The BS allocates channel resources at the end of the corresponding request interval. Video terminals have higher priority in acquiring the slots they demand. In the case that a new VF arrives and the number of ftp slot reservations is such that the video terminal can not be fully serviced, the BS preempts ftp reservations in favor of video terminals waiting for transmission. If a full allocation to the video terminal is still not possible, the BS grants to the video terminals as many of the slots they requested as possible (i.e., the BS makes a partial allocation). The BS allocates the earliest available information slots to the video terminals, which, if needed, keep these slots in the following channel frames, until the next video frame (VF) arrives. 
The channel rate is $20 \mathrm{Mbps}$ (from [6]), and the frame duration equal to $12 \mathrm{~ms}$ (from [2]). The $12 \mathrm{~ms}$ of frame duration accommodate 566 slots (556 information slots plus 10 request slots).

\section{Error Model}

We use a simplified Fritchman Markov model (from [7]) to emulate the process of packet transmission errors. The Markov model used (presented in Figure 1) comprises of 6 states. State $s_{0}$ represents the "good state" and all other states represent the "bad states". When the channel is in state $s_{0}$, it can either remain in this state or make the transition to state $s_{1}$ (with probability $p_{0}$ ). When the channel is in a bad state, the transition is either to the next higher state or back to state $s_{0}$, based on the status of the currently received packet. This means that the channel does not remain in any of the "bad states" for more than 1 slot. With this model, it is only possible to generate burst errors of length equal to 5 slots at most. The transition probabilities $\left(\mathrm{p}_{0}, \mathrm{p}_{1}, \ldots \mathrm{p}_{5}\right)$ of the error model are $(0.0000446,0.100324,0.164083,0.149606,0.526316,0)$, respectively. The probability that the channel is in a good state is $\mathrm{p}_{\text {good }}=0.99995$, and the total probability for a transition from a bad state to the good state is $\mathrm{p}_{\text {bad-good }}=0.8924$.

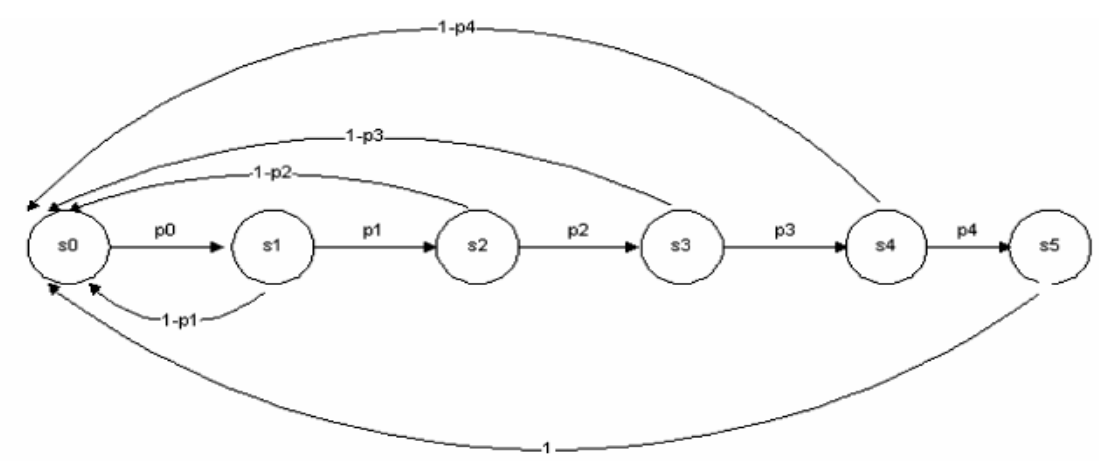

Fig. 1. Error Model

\section{Simulation Results and Discussion}

Via an extensive simulation study we studied system behavior and performance under all possible movie loads from 1 to 6 movies. For each load, all different combinations were examined (63 in total), from 10 times each (Monte Carlo method).

Table 1 shows the average results of the simulation runs under all possible movie loads. As the number of video terminals increases, the aggregate bit rate gets smoother, since the superposition of the movies is known from the literature to always be less bursty than a single movie, and the average bandwidth of the superposition is close to the sum of the mean bit rates of the movies. This is proven once more in our study, as the case of the system accommodating only one movie is shown to provide the smallest throughput than any other case of movie load. The $\lambda$ parameter corresponds to the maximum ftp session 
Table 1. Integration Results for the superposition of movies

\begin{tabular}{|c|c|c|}
\hline System Load & $\begin{array}{c}\text { Average } \lambda \\
\text { (ftp sessions/frame) }\end{array}$ & $\begin{array}{c}\text { Average Channel } \\
\text { Throughput }(\%)\end{array}$ \\
\hline 1 Movie & 0.312 & 81.32 \\
\hline 2 Movies & 0.272 & 81.99 \\
\hline 3 Movies & 0.207 & 83.64 \\
\hline 4 Movies & 0.158 & 88.75 \\
\hline 5 Movies & 0.087 & 95.28 \\
\hline 6 Movies & 0.019 & 93.54 \\
\hline
\end{tabular}

arrival rate (expressed in sessions per frame) sustained by the system (i.e., such that the video packet dropping probability $<0.0001$ and the average ftp session delay $<1$ minute). Our results show that the proposed mechanism achieves very high aggregate channel throughput (steadily more than 80\%) for all cases of combined video traffic load, while preserving the Quality of Service (QoS) requirements of both video and ftp traffic types.

The reason that the channel throughput decreases when six videos are active compared to the case of five active videos, can be explained if we sum up the peak bit rates of the six movies; the total peak rate reaches $25 \mathrm{Mbps}$, which is significantly higher than the total channel capacity. Of course, it is rather rare that all of the 6 video terminals would transmit at their peak bit rates at the same time but still their superposition surpasses for a few video frames the channel capacity. This forces the system to experience, in these video frames, severe video packet dropping, because of the very strict video dropping probability requirements, and therefore leads to the decrease in throughput in comparison with the 5 active video terminals scenario.

\section{References}

1. [Online] http://peach.eas.asu.edu/index.html

2. P. Koutsakis, S. Psychis and M. Paterakis, "On the Integration of MPEG-4 Video Streams with Voice and E-mail Data Packet Traffic over Wireless Picocellular Networks", in Proceedings of the IEEE PIMRC 2001 Conference, San Diego, California, USA.

3. P. Tran-Gia, D. Staehle, K. Leibnitz, "Source traffic modeling of wireless applications", International Journal of Electronics and Communications, Vol. 55, No. 1, 2001, pp. 27-37.

4. "Universal Mobile Telecommunication System (UMTS); Selection Procedures for the Choice of Radio Transmission Technologies of the UMTS", Technical Report TR 101112 v3.2.0, ETSI, April 1998.

5. A. Cleary and M. Paterakis, "Design and Performance Evaluation of an RRA Scheme for Voice-Data Channel Access in Outdoor Microcellular Wireless Environments", ACM/Baltzer MONET Journal, Vol. 2, No.1, pp. 31-43, 1997.

6. N. Passas, D. Skyrianoglou and L. Merakos, "Traffic Scheduling in Wireless ATM Networks", in Proceedings of the IEEE ATM'97 Workshop, Lisbon, Portugal, May 1997.

7. C. Y. Hsu, A. Ortega and M. Khansari, "Rate Control for Robust Video Transmission over Burst-Error Wireless Channels", IEEE Journal on Selected Areas in Communications, Vol. 17, No.5, May 1999. 